Ingrid Oliveira de Nunes

\title{
User-centric Preference-based Decision Making
}

Thesis presented to the Programa de Pós-Graduação em Informática of the Departamento de Informática, PUC-Rio as partial fulfillment of the requirements for the degree of Doutor em Informática.

Advisor: Prof. Carlos José Pereira de Lucena 


\section{Ingrid Oliveira de Nunes}

\section{User-centric Preference-based Decision Making}

Thesis presented to the Programa de Pós-Graduação em Informática, of the Departamento de Informática do Centro Técnico Científico da PUC-Rio, as partial fulfillment of the requirements for the degree of Doutor.

Prof. Carlos José Pereira de Lucena
Advisor
Departamento de Informática - PUC-Rio

Prof. Simone Diniz Junqueira Barbosa

Departamento de Informática - PUC-Rio

Prof. Hugo Fuks

Departamento de Informática - PUC-Rio

Prof. Rafael Heitor Bordini

PUC/RS

Prof. Jaime Simão Sichman

USP

Prof. José Eugenio Leal

Coordinator of the Centro Técnico Científico da PUC-Rio

Rio de Janeiro, September 20, 2012 
All rights reserved.

\section{Ingrid Oliveira de Nunes}

She has obtained the Master degree in Informatics at the Pontifical Catholic University of Rio de Janeiro (PUC-Rio), in 2009, Rio de Janeiro, Brazil, and received the degree of Bachelor in Computer Science at the Federal University of Rio Grande do Sul (UFRGS), in 2006, Porto Alegre, Brazil. During her Ph.D., she visited the University of Waterloo (Canada), with two three-month research visits, and King's College London (UK) for one year, as part of the CNPq Sandwich Ph.D. Programme. She also worked as a software developer at the e-Core Desenvolvimento de Software company from 2005 to 2007 . Her main research interests are multi-agent systems, decision making, preference reasoning and agent-oriented software engineering.

Bibliographic data

Nunes, Ingrid Oliveira de

User-centric Preference-based Decision Making / Ingrid Oliveira de Nunes; orientador: Carlos José Pereira de Lucena. - 2012.

298 f.: il. ; $30 \mathrm{~cm}$

1. Tese (doutorado) - Pontifícia Universidade Católica do Rio de Janeiro, Departamento de Informática, 2012.

Inclui bibliografia

1. Informática - Teses. 2. Tomada de Decisão. 3. Representação de Preferências. 4. Raciocínio sobre Preferências. 5. Explicação a Usuários. 6. Raciocínio Humano. 7. Sistemas de Suporte à Decisão. I. Lucena, Carlos José Pereira de. II. Pontifícia Universidade Católica do Rio de Janeiro. Departamento de Informática. III. Título. 
To my parents, Daltro and Suzana.

To my brothers, Gustavo and Matthias.

I love you all. 


\section{Acknowledgments}

I must first express my gratitude towards my supervisor, Prof. Carlos Lucena, who is one of the leading Computer Science researchers in Brazil. It was a privilege to have the opportunity to work with him during my master and Ph.D. His guidance was essential to complete this thesis and make me an independent researcher. I also give my sincere thanks to Prof. Michael Luck and Dr. Simon Miles, my supervisors during the sandwich Ph.D. period at King's College London. I am grateful for their insightful comments both in my work and in this thesis, for their support, and for many motivating discussions.

I would like to thank the other members of my examining committee, Prof. Rafael Bordini, Prof. Jaime Sichman, Prof. Simone Barbosa and Prof. Hugo Fuks, for taking the time of reading my thesis and giving me constructive feedbacks. Prof. Simone also contributed to this thesis with fruitful discussions, and had an important role in the work that was the seed of this thesis. Thanks to Prof. Don Cowan for his feedback and making my research visits to University of Waterloo possible.

I would also like to thank all my friends and colleagues from PUC-Rio and King's College London for their help, support and friendship. I will mention some of them, and I apologise for those not explicitly mentioned. Many thanks to Elder Cirilo for our engaging joint work, discussions, and friendship. The research visits to UW would not have been so productive and fun without him. Thanks to Camila Nunes, Francisco Dantas and Isela Macia, who started their Ph.D. at the same time as me, and became wonderful friends and colleagues. A special thanks also goes to Vera Menezes for her friendship and taking care of me during this journey in Rio. Daniele Nantes, Luis Fernando Dalla Santa, and Lina Barakat, thank you for making my period in London so nice. My friends from Porto Alegre, Ana Maria Souza, Daniela Malvasio, Graziela Becker and Paula Kruger, thank you for always being there. I also thank Prof. Carlos Lisbôa and Prof. Maria Lúcia Lisbôa for their friendship and for having encouraged me in some of my important achievements.

I am and will always be extremely grateful to my beloved parents, Daltro Nunes and Suzana Nunes, who raised me and taught me to give priority in my life to the quest for knowledge. Thanks for their love and support throughout my studies and work, and being examples of the kind of person I want to be. I also thank my brothers, Gustavo Nunes and Matthias Nunes, for being so supportive.

In conclusion, I recognise that this research would not have been possible without the financial assistance of CNPq, FAPERJ and CAPES, and infrastructure of the Pontifical Catholic University of Rio de Janeiro (PUC-Rio) and King's College London, and express my gratitude to those agencies and universities. 


\section{Resumo}

Nunes, Ingrid Oliveira de; Lucena, Carlos José Pereira de. Tomada de Decisão baseada em Preferências e centrada no Usuário. Rio de Janeiro, 2012. 298p. Tese de Doutorado - Departamento de Informática, Pontifícia Universidade Católica do Rio de Janeiro.

A escolha de uma entre um conjunto de opções disponível normalmente requer a resolução de trade-offs, contudo esperar que as pessoas avaliem cada uma das opções de um grande conjunto pode ser inviável devido ao tempo e ao esforço cognitivo necessários para realizar tal análise, fazendo com que elas fiquem freqüentemente insatisfeitas com suas escolhas. Sistemas de software podem dar suporte à tomada de decisão humana ou mesmo automatizar esse processo, entretanto existem muitos desafios que estão associados com o oferecimento de tal suporte. Esta tese lida, em particular, com três destes desafios: (i) como representar preferências dos usuários; (ii) como raciocinar sobre estas preferências e tomar decisões; e (iii) como justificar tais decisões. Diferentes abordagens têm sido propostas para a representação e raciocínio sobre preferências qualitativas, mas estas abordagens lidam com um conjunto restrito de tipos de preferências, e portanto não são capazes de processar preferências fornecidas por usuários em muitos cenários realistas. Nesta tese, apresentam-se três principais contribuições. A primeira delas consiste de um novo metamodelo de preferências, o qual foi desenvolvido de acordo com um estudo sobre a expressão de preferências, permitindo a representação de preferências em alto-nível. Segundo, uma nova técnica de tomada de decisão automatizada é proposta, a qual escolhe uma opção de um conjunto de opções disponível baseada em preferências expressas em uma linguagem construída de acordo com o metamodelo proposto, explorando termos da linguagem natural, tais como atos de fala expressivos. A técnica vai além das preferências fornecidas para tomar a decisão através da incorporação de princípios da psicologia, que focam como os humanos tomam decisões, já que as preferências fornecidas tipicamente não são suficientes para resolver trade-offs entre as opções disponíveis. Terceiro, apresenta-se uma técnica de geração de explicação, que utiliza modelos construídos pela técnica de tomada de decisão para justificar escolhas, e segue diretrizes e padrões que foram derivados de um estudo sobre explicações a respeito de escolhas, também realizado no contexto desta tese. Um estudo com usuários foi feito para avaliar a abordagem, o qual mostra que (i) a linguagem de preferências é adequada para usuários expressarem suas preferências, que (ii) a técnica de tomada de decisão faz escolhas que os usuários consideram de alta qualidade, e que (iii) as explicações fornecidas permitem que usuários entendam por que a escolha foi feita, bem como melhora a confiança na decisão tomada. 


\section{Palavras-chave}

Tomada de Decisão. Representação de Preferências. Raciocínio sobre Preferências. Explicação a Usuários. Raciocínio Humano. Sistemas de Suporte à Decisão. 


\section{Abstract}

Nunes, Ingrid Oliveira de; Lucena, Carlos José Pereira de. User-centric Preference-based Decision Making. Rio de Janeiro, 2012. 298p. DSc Thesis - Departmento de Informática, Pontifícia Universidade Católica do Rio de Janeiro.

Choosing from a set of available options often requires resolution of trade-offs but it can be unfeasible for humans to carefully evaluate each option of a large set due to the required time and cognitive effort. Consequently, they are often unsatisfied with their choices. Software systems can support human decision making or even automate this process, but there are many challenges associated with the provision of such support. In this thesis we deal in particular with three of them: (i) how to represent user preferences; (ii) how to reason about preferences and make decisions; and (iii) how to justify such decisions. Different approaches have been proposed for representing and reasoning about qualitative preferences, but they address a restricted set of preference types, and therefore are not able to process preferences provided by users in many realistic scenarios. This thesis provides three main contributions. First, we introduce a new preference metamodel founded on a study of how humans express preferences, allowing the representation of high-level preferences. Second, we propose an automated decision making technique, which chooses an option from a set available based on preferences expressed in a language based on our metamodel, exploiting natural-language terms. Our technique goes beyond the provided preferences to make a decision with the incorporation of psychology principles, which concern how humans make decisions, as the provided preferences are typically not enough to resolve trade-offs among available options. Third, we present an explanation generation technique, which uses models built by our decision making technique to justify choices, and follows guidelines and patterns that we derived from a study of choice explanation. A user study was performed to evaluate our approach, which shows that (i) our preference language is adequate for users to express their preferences, (ii) our decision making technique makes choices that users consider as having good quality, and (iii) the provided explanations allow users to understand why the choice was made and improves the confidence in the decision.

\section{Keywords}

Decision making. Preference Representation. Preference Reasoning. User Explanations. Human Reasoning. Decision Support Systems. 


\section{Table of Contents}

1 Introduction 19

1.1 Problem Statement and Limitations of Existing Work 21

1.2 Proposed Solution and Contributions Overview 23

1.3 outline 24

I Preference Representation 26

2 Understanding User Ability to Express Preferences 28

2.1 Study Description 29

2.1 .1 Research Questions 30

2.1 .2 Procedure 32

2.1 .3 Participants 34

2.2 Results and Analysis $\quad 35$

2.3 Discussion 49

2.3.1 Supporting the Preference Expression 51

2.3.2 Providing Different Forms of Expressing Preferences 51

2.4 Final Remarks 56

3 Preterence Metamodel 57

3.1 Ontology Metamodel 57

3.2 Propositional Formulae $\quad 60$

3.3 Preference Metamodell 62

3.3 .1 Overview 62

3.3.2 Simple Preferences 63

3.3 .3 Preference Statements 64

3.3 .4 Preterence Priority 66

3.3.5 Interaction among Preterences and Targets 66

3.4 Final Remarks 68

$4 \quad$ Related Work on Preference Representation 69

4.1 Constraint-based Approaches 69

4.1 .1 Soft-constraints 69

4.1.2 Preference-based Problem Solving for Constraint Programming 71

$\begin{array}{lll}4.2 \text { Graphically-structured Approaches } & 71\end{array}$

4.2.1 CP-nets: Conditional Ceteris Paribus Preterence Statements 71

4.2 .2 TCP-nets: Modelling of Preference and Importance 72

4.3 Database Approaches 73

4.3 .1 Scoring Function 73

4.3 .2 Preference Formulae in Relational Queries 74

4.3.3 Foundations of Preferences in Database Systems 75

4.3.4 Personalisation of Queries based on User Preterences 76

4.4 Semantic Web Approaches 79

4.4.1 OWLPret: a Declarative Preference Representation 79 
$\begin{array}{lll}4.4 .2 & \text { Metamodelling Approach to Preference Management } & 80\end{array}$

4.4 .3 Situated Preferences for Personalised Database Applications 81

4.5 Non-parametric Representation of User Preferences 81

4.6 Comparison of Preference Representation Models 82

4.7 Final Remarks 85

II Preference Reasoning $\quad 86$

5 A Systematic Review of Reasoning about Preferences $\quad \mathbf{8 8}$

5.1 Review Method 89

5.2 Background on Multi-Attribute Utility Theory 91

5.3 Utility Function-based Approaches 94

5.3.1 CUlnetworks 94

$\begin{array}{lll}5.3 .2 & \text { Utility functions for Ceteris Paribus Preferences } & 96\end{array}$

$\begin{array}{lll}5.3 .3 & \text { Learning Utility Functions with SVM } & 98\end{array}$

$\begin{array}{llr}5.4 & \text { Constraint Programming } & 99\end{array}$

$\begin{array}{lll}5.4 .1 & \text { Semiring-based Constraint Satisfaction } & 100\end{array}$

5.4 .2 Preference-based Problem Solving for Constraint Programming 103

$\begin{array}{lll}5.5 & \text { Graphically-structured Approaches } & 105\end{array}$

$\begin{array}{lll}5.5 .1 \text { CP-nets } & 105\end{array}$

$\begin{array}{lll}5.5 .2 & \text { Combining CP-nets and Soft Constraints } & 108\end{array}$

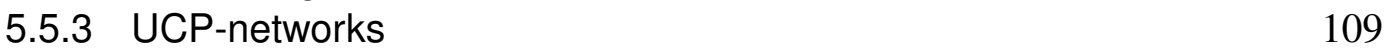

5.5 .4 TCP-nets 110

5.5.5 Graphically Structured Value Function Compilation 113

5.6 Query-based Approaches 114

$\begin{array}{lll}5.6 .1 & \text { Scoring Function } & 115\end{array}$

$\begin{array}{ll}5.6 .2 \text { Winnow } & 116\end{array}$

5.6.3 Best-Matches-Only Query Model 118

5.6.4 Query Personalisation based on Preferences 119

$\begin{array}{lll}5.6 .5 & \text { OWLPref } & 120\end{array}$

5.7 Preferences in Argumentation Frameworks 123

$\begin{array}{lll}5.8 \text { Discussion } & 125\end{array}$

$\begin{array}{lll}5.9 & \text { Final Considerations } & 129\end{array}$

6 An Automated Decision Maker with User-centric Principles 131

$\begin{array}{lll}6.1 & \text { Scope and Assumptions } & 131\end{array}$

6.2 Preference Language and Running Example 132

\begin{tabular}{lll}
\hline 6.3 & lechnique Overview & 135
\end{tabular}

$\begin{array}{llr}6.4 & \text { Pre-processing } & 139\end{array}$

$\begin{array}{ll}6.4 .1 & 139\end{array}$

$\begin{array}{lll}6.4 .2 & \text { Options-Attribute Preference Model } & 143\end{array}$

$\begin{array}{lll}6.5 & \text { Explication } & 148\end{array}$

$\begin{array}{ll}6.5 .1 & \text { Upper bound } \\ 6.5 .2 & 150\end{array}$

6.5.2 Lower bound 150

6.5 .3 Around 151

$\begin{array}{lll}6.5 .4 & \text { Interval } & 151\end{array}$

$\begin{array}{lll}6.6 & \text { Elimination } & 153\end{array}$

6.6.1 Eliminating Dominated Options 153 
$\begin{array}{lll}6.6 .2 & \text { Applying Cut-off Values } & 154\end{array}$

6.7 Selection 156

$\begin{array}{lll}6.7 .1 & \text { Cost-benefit Analysis } & 157\end{array}$

6.7 .2 Trade-off Contrast 170

6.7 .3 Extremeness Aversion 171

6.7.4 The Decision Function: Comparing Relative Option values 173

6.8 Comparison with Related Work and Evaluation 177

$\begin{array}{ll}6.9 \text { Final Remarks } & 181\end{array}$

III User Explanation 182

$7 \quad$ Background on Explanation Approaches $\quad 184$

7.1 Expert Systems: the Roots of Explanation 184

7.2 Explanation in Recommender Systems 187

$\begin{array}{lll}7.3 & \text { Explanations for Over-constrained Problems } & 189\end{array}$

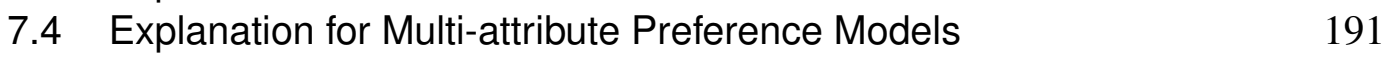

$\begin{array}{lll}7.5 & \text { Final Remarks } & 193\end{array}$

8 Guidelines and Patterns for Explanations 195

8.1 Study Description 195

8.1.1 Research Questions 196

$\begin{array}{ll}8.1 .2 & 197\end{array}$

$\begin{array}{lll}8.1 .3 & \text { Participants } & 199\end{array}$

$\begin{array}{lll}8.2 & \text { Results and Analysis } & 200\end{array}$

8.3 Interpretation 210

$\begin{array}{lll}8.3 .1 & \text { Explanation for Choice } & 210\end{array}$

8.3.2 Explanation for Rejection 211

8.4 Guidelines and Patterns 212

8.4.1 Guidelines 212

8.4 .2 Patterns 213

8.5 Final Considerations 220

9 Generating Explanations to Justify Choice $\quad 221$

$\begin{array}{lll}9.1 \text { Notation } & 221\end{array}$

9.2 Explanation Parameters: Selecting Relevant Attributes 222

9.2.1 Single-attribute Selection 222

9.2 .2 Multi-attribute Selection 225

9.3 Choosing and Generating an Explanation 230

9.4 The Apartment Example: Illustrating our Approach 232

9.5 Comparison with Related Work and Performance Evaluation 235

$\begin{array}{lll}9.6 & \text { Final Considerations } & 237\end{array}$

IV Final 239

10 Evaluating our Approach with a User Study 240

$\begin{array}{ll}10.1 \text { Study Description } & 240\end{array}$

$\begin{array}{ll}\text { 10.1.1 Research Questions and Hypotheses } & 241\end{array}$

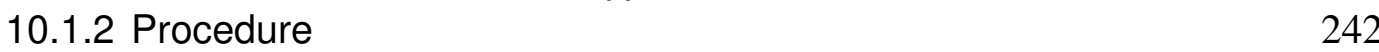


$\begin{array}{ll}\text { 10.1.3 Participants } & 245\end{array}$

$\begin{array}{ll}10.2 \text { Results and Analysis } & 246\end{array}$

10.2.1 Preferences and Language Evaluation 246

10.2.2 Choice Evaluation and Explanation Impact 250

$\begin{array}{ll}10.2 .3 \text { Explanation Comparison } & 253\end{array}$

\begin{tabular}{ll} 
10.2.4 Approach Evaluation 258 & 253 \\
\hline & 26.3
\end{tabular}

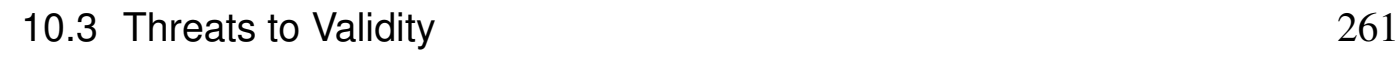

$\begin{array}{ll}10.4 \text { Final Remarks } & 261\end{array}$

11 Conclusion $\quad 263$

$\begin{array}{ll}11.1 \text { Contributions } & 264\end{array}$

$\begin{array}{ll}11.2 \text { Future Work } & 266\end{array}$

$\begin{array}{lr}\text { Bibliography } & 269\end{array}$

A Questionnaire: Preference Expression 279

$\begin{array}{lll}\text { A.1 Introduction: Survey about User Preferences } & 279\end{array}$

$\begin{array}{ll}\text { A.2 PartI: User Data } & 279\end{array}$

$\begin{array}{lll}\text { A.3 Part II: Preference Specification } & 280\end{array}$

\begin{tabular}{lll}
\hline A.4 Part III: Options Selection & 280
\end{tabular}

A.5 Part IV: Preference Specification Review 281

B LSpecification $\quad 283$

B.1 Ontology Metamodel 283

B.1.1 Concept and Attributes 283

B.1.2 Instantiation 285

$\begin{array}{lll}\text { B.2 } & \text { Propositionalformulae } & 287\end{array}$

B.3 Preference Metamodel 288

$\begin{array}{ll}\text { B.3.1 Preference } & 289\end{array}$

\begin{tabular}{ll}
$B .3 .2$ & Priority \\
\hline
\end{tabular}

C Questionnaire: Survey on Reasons for Choice 292

C.1 Introduction: Survey on Reasons for Choice 292

C.2 Partl: User Data 292

C.3 Part II: Option Selection 293

$\begin{array}{lll}\text { C.4 Part III: Reasons for your Choice } & 293\end{array}$

D Application for Evaluating our Proposed Approach 295 


\section{List of Figures}

1.1 Thesis components and their relationship. 25

2.1 Nature of Preference Changes. 39

2.2 Preference Changes x Domain Knowledge (Percentage). 40

2.3 Preference Specification Analysis. 41

2.4 Preference Specification Types (percentage). 43

2.5 Preference Specification Characteristics (percentage). 44

3.1 Ontology metamodel. 58

$\begin{array}{lll}3.2 & \text { Ontology primitive types. } & 59\end{array}$

$\begin{array}{lll}3.3 & \text { Propositional formula model. } & 60\end{array}$

3.4 Overview of the preference metamodel. $\quad 62$

3.5 Goals and Constraints. 63

3.6 Don't care preference. $\quad 64$

3.7 Preference targets.

3.8 Preference statements model. 65

3.9 Preference priority model. 66

4.1 Personalisation Graph (Koutrika and loannidis 2006). $\quad 78$

4.2 Metamodels (Tapucu et al. 2008).

5.1 Example of a CP-net (Boutilier et al. 2004). 106

6.1 Technique Overview. 137

6.2 Modifier scale. 142

$\begin{array}{lll}6.3 & \text { Calculating node values. } & 159\end{array}$

6.4 Tagging an attribute domain associated with a goal. 164

6.5 Attribute weights calculated with the logarithmic function. 169

6.6 Expert vs. our technique: first choices. 179

6.7 Expert vs. our technique: up to five choices. 180

8.1 Hotel Choice. 201

8.2 Explanation types used to justify each chosen hotel. 203

8.3 Rejection explanation types. 206

10.1 Preference Analysis. 248

10.2 Language Evaluation. 249

10.3 Choice Evaluation and Explanation Impact. 251

10.4 Explanation Impact — Iransparency. 253

10.5 Explanation Comparison (I). 254

10.6 Explanation Comparison (II). 255

10.7 Approach Evaluation (I). 259

10.8 Approach Evaluation (II). 260

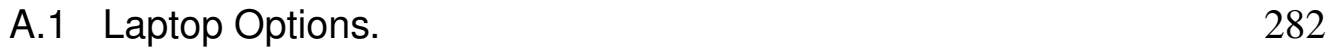

\begin{tabular}{lll}
\hline C.1 Hotel Options. & 294 \\
\hline
\end{tabular} 
D.1 Participant Data. 


\section{List of Tables}

2.1 Goal Definition (GQM template). 29

2.2 Research questions and their evaluation approach. 31

2.3 Qualitative and quantitative data collected. 34

2.4 Demographic Characteristics of Participants. 35

2.5 Domain Specialist Recommendation - Matches per Group of $\begin{array}{ll}\text { Participants. } & 37\end{array}$

2.6 Domain Specialist Recommendation - Position Matched. 37

2.7 Preference Changes. 38

2.8 Preference Types vs. Domain Specialist Recommendation. 46

2.9 Time Taken for Specifying Initial Preferences. 47

2.10 Number of Chosen Laptops. 48

2.11 Number of Steps Taken to Choose Laptops. 49

2.12 Expressive speech acts adopted by participants in assessment $\begin{array}{ll}\text { statements. } & 52\end{array}$

$3.1 \quad$ Examples of preferences and priorities. 67

4.1 Base Preference Constructors. 76

4.2 Types of AttributeValuePreference. $\quad 79$

4.3 Comparison of Preference Representation Approaches (1). 83

4.4 Comparison of Preference Representation Approaches (2). $\quad 84$

5.1 Main terms adopted in decision making. 92

5.2 Different specific trameworks modelled as c-semirings (Meseguer et al. 2006). 101

5.3 Complexity Analysis of CP-nets (boolean attributes). 107

5.4 Comparison among Approaches to Reason about Preferences. 127

6.1 Preference language. 133

$\begin{array}{lll}6.2 & \text { Available apartments. } & 135\end{array}$

6.3 PSM of the Apartment Decision Problem. 143

6.4 Index used to compare PSM values. 145

6.5 OAPM of the Apartment Decision Problem. 149

6.6 Updated OAPM of the Apartment Decision Problem. 153

6.7 Cost-benefit Analysis for the Apartment Decision Problem. 166

6.8 Trade-off Analysis for the Apartment Decision Problem. 171

6.9 Options for Illustrating the Impact of the User-centric Principles. 174

6.10 Options for Illustrating the Impact of Trade-off Contrast. 175

6.11 Decision Function of the Apartment Decision Problem. 176

6.12 Complexity Analysis of our Technique. 177

6.13 Reasoning Approaches vs. Preferences. 178

6.14 Analysis of Domain Expert and our Technique choices. 181

\begin{tabular}{|lll}
\hline .1 & Toulmin's Argument Structure. & 187
\end{tabular}

7.2 Aims of Recommender Systems (Tintarev and Masthoff 2007). 188

7.3 Labreuche's Approach Examples. 193 
8.1 Goal Definition (GQM template). 196

8.2 Research questions and their evaluation approach. 197

8.3 Data collected in our study. 199

8.4 Demographic Characteristics of Participants. 200

8.5 Example of Justification for Acceptance. 202

8.6 Main explanation types used tor justifying hotels of the G-3 group. 204

8.7 Example of Justification for Rejection. 205

8.8 Results for additional characteristics observed in justifications. 209

9.1 Trade-off explanations: selection of pros and cons to be shown. 231

9.2 Explanation Types. 232

9.3 Comparison of selected decisive criteria. 236

9.4 Explanation Evaluation. 237

10.1 Goal Definition (GQM template). 241

10.2 Measured Variables - adapted from (Chen and Pu 2010). 245

10.3 Demographic Characteristics of Participants. 246

10.4 Choice evaluation and explanation impact measurements. 252

10.5 Examples illustrating the main differences between Klein and Shortlitfe's approach and ours. 256

10.6 Explanation comparison measurements. 257

10.7 Approach evaluation measurements. 258 


\section{Abbreviation and Acronym List}

AI artificial intelligence

AMVF additive multi-attribute value function

AVPO attribute value partial order

BMO Best-Matches-Only

CSP Constraint Satisfaction Problem

CIT conditional importance table

CPT conditional preference table

DAG directed acyclic graph

EAF Extended Argumentation Framework

EBNF Extended Backus Naur Form

ES Expert System

GA generalised additive

GAI generalised additive independence

GEA Generator of Evaluative Arguments

GQM goal-question-metric

IVA Interpretive Value Analysis

MAUT Multi-Attribute Utility Theory

OAPM Options-Attribute Preference Model

OVF optimal value function

PSM Preference Satisfaction Model

SCSP Soft Constraint Satisfaction Problem

SE Software Engineering

SLO Soft-constraint Lexicographic Ordering 
SVM Support Vector Machines

UML Unified Modeling Language

UF utility function 